\title{
Tidal Influences on Cluster Dwarf Evolution
}

\author{
R. Smith ${ }^{1}$, J. I. Davies ${ }^{1}$ and A. Nelson ${ }^{1}$ \\ ${ }^{1}$ School of Physics \& Astronomy, University of Wales, Cardiff, UK
}

\begin{abstract}
Using N-Body/Treecode,SPH simulations, including a self regulating Star Formationfeedback model, the influence of cluster tidal forces on infalling dwarf irregulars is investigated. Results suggest that an encounter with the cluster potential can disrupt a rotating disk causing morphological transformation of the gaseous component, resulting in an enhancement of star formation rates (SFRs) of up to an order of magnitude.
\end{abstract}

\section{Modelling Super-novae feedback in galaxies}

The code, gf, uses N-body/Treecode calculations of gravity and SPH to calculate fluid quantities. SFRs are governed by a Schmidt-law. A new model allows star formation feedback to effectively self regulate SFRs by providing gas particles with a thermal energy resevoir, and separate turbulent energy resevoir. Initially super-novae(SN) feedback energy is deposited as turbulent motion in gas providing additional pressure support, before the turbulence decays into thermal energy where it can be radiated away.

By this means high density regions within the gas generate high SFRs and are accompanied by strong SN feedback, increasing the velocity dispersion of the gas. In turn densities are lowered and SFRs are regulated.

The model is tuned using a simulation of as late type spiral galaxy(see figure1(a)), modelled with a live NFW dark matter halo $\left(10^{12} M_{\odot}\right)$, and an exponential disk of gas $(20 \%)$ and stars $(80 \%)$. In such a galaxy there are tight empirical constraints on SFRs (Kennicutt, 1998), and on velocity dispersion variations within the disk (Low, 2004) from 4-15 $\mathrm{kms}^{-1}$ (see figure1(b)) where the chief driving mechanism is thought to be SN feedback. The star formation threshold - the disk radius at which star formation ceases - is reproduced using a specific volume density cutoff (Schaye, 2004).

\section{Modelling Dwarfs Galaxies in the Cluster}

Dwarf irregulars(dIrrs) are modelled as gas rich $(\geqslant 80 \%)$ exponential disks within live dark matter haloes $\left(10^{10} M_{\odot}\right)$. Application of the full model to an isolated, field dwarf irregular produces smooth, low column density disks with accordingly low star formation rates (see figure $2(\mathrm{a}) \& 2(\mathrm{~b})$ ).

The Virgo cluster potential is modelled using a static plummer potential with a mass $10^{14} M_{\odot}$. dIrrs are modelled as an infalling population, hence highly non radial orbits plunge the dIrrs deep in the core of the cluster (pericluster distance $\sim 100 p c$ ).

During the infall there is little enhancement of star formation as the dIrr effectively free falls in the cluster potential, and tidal forces are small due to the smooth nature of the potential and small diameter of the disk. However on passing the cluster core, strong tidal forces disrupt the disk as rotational angular momentum about the galaxy's centre is transfered into translational angular momentum about the cluster core. The result is disk collapse with a burst of star formation (see figure $3(\mathrm{a})$ \& (b)), eventually resulting in a 

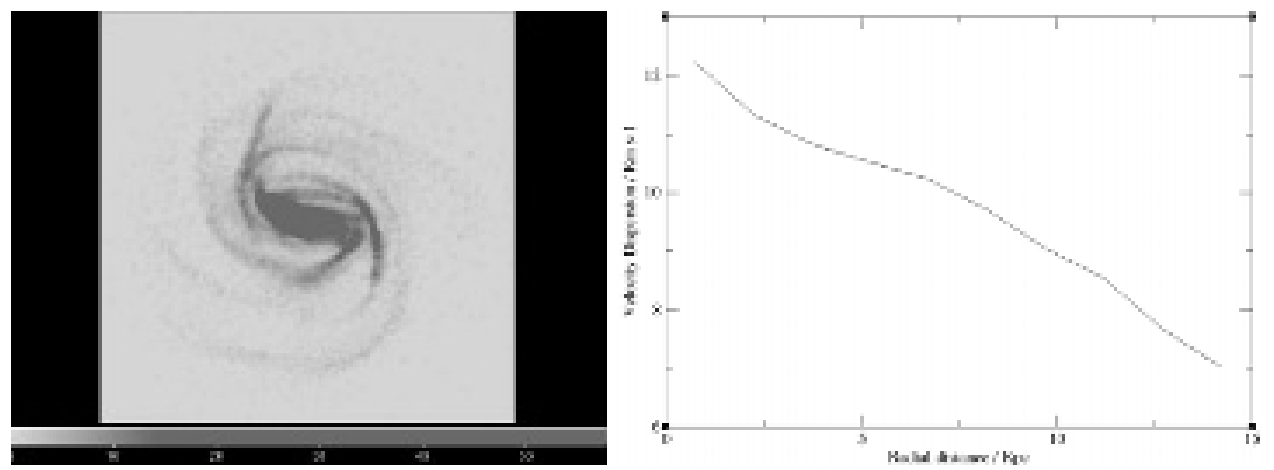

Figure 1. (a)Column density of late type spiral, $M_{\odot} p c^{-2}$,(b)Velocity dispersion across the disk, $\mathrm{kms}^{-1}$
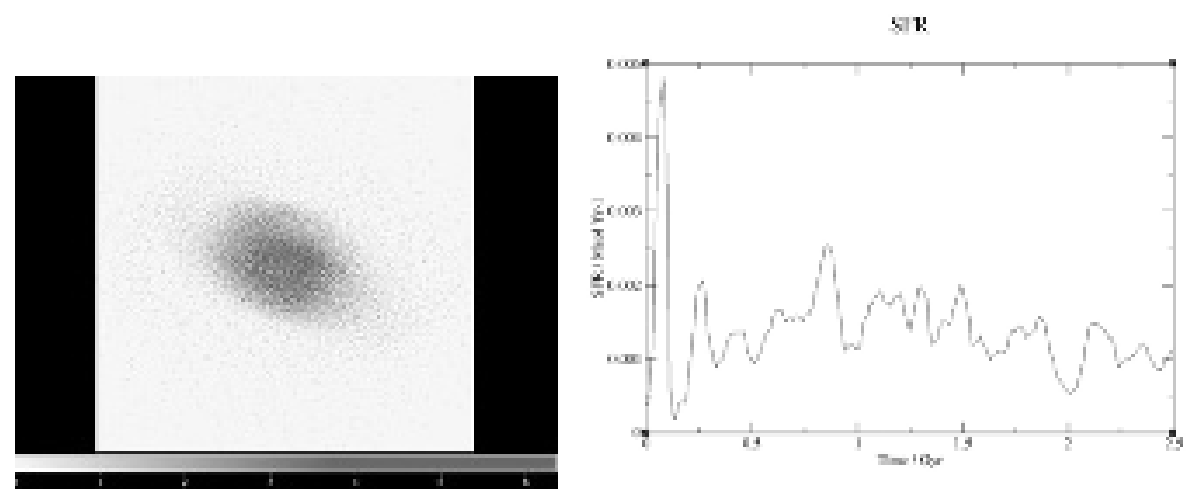

Figure 2. (a)Column density of $\mathrm{dIrr}, M_{\odot} p c^{-2},(\mathrm{~b}) \mathrm{SFR}$ of isolated $\mathrm{dIrr}, M_{\odot} y r^{-1}$
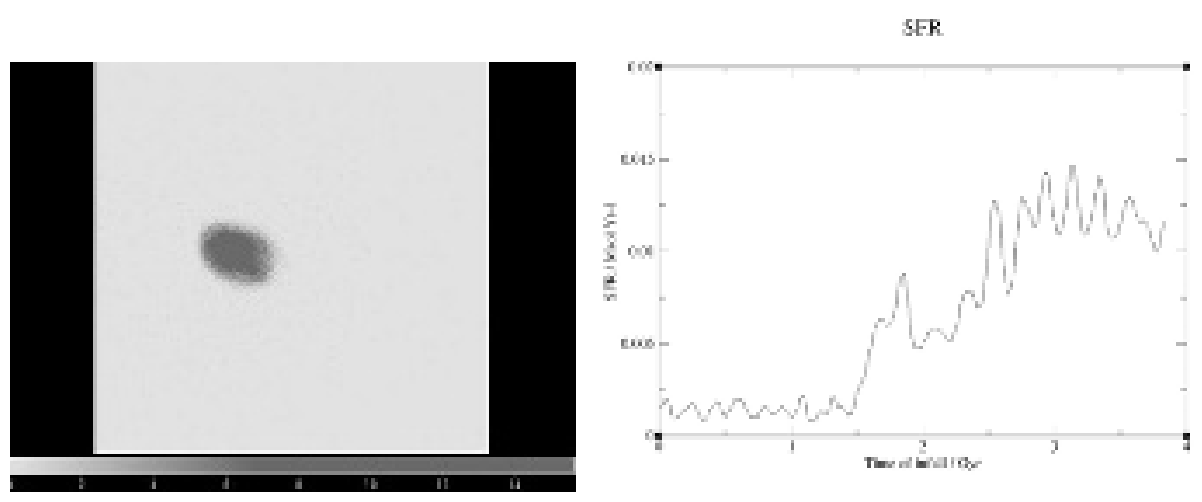

Figure 3. (a)Column density of disrupted dIrr, $M_{\odot} p c^{-2}$, (b)SFR of infalling dIrr, $M_{\odot} y r^{-1}$

spheroidal gas component with a continuous high star formation rate, only regulated by strong SN feedback. Resulting SFRs are in good agreement with observed values derived using $\mathrm{H} \alpha$ observations of Virgo cluster dIrrs (www.goldmine.mib.infn.it). 


\section{Conclusions \& further work:}

Results suggest that strong tidal forces in the Virgo cluster core can strongly disrupt the rotation of dIrr disks - a rotation that is necessary to maintain low column densities and SFRs. The interaction with the cluster potential drives disk material radially inwards producing a spheroidal gas component with high SFRs. However this conclusion cannot be complete without accounting for ram pressure stripping within the cluster core, a mechanism that is likely to be highly effective at removing large quantities of gas from the dIrr. This could shorten or even negate a tidally induced star formation burst by removing the fuel for star formation. Current investigations involve including the ram pressure stripping mechanism into simulations (Vollmer, 2001), resolving the cluster substructure's infuence on the global potential field, and in turn its effect on infalling dIrrs.

\section{References}

Kennicutt, R.C. 1998, ARAA, 36, 189

Low, M-M.M. 2004, Ap\&SSS, 289, 323

Schaye, J. 2004, ApJ, 609,667

Vollmer, B, et al 2001, ApJ, 516, 708 